\title{
Niños que leen, niños que comprenden intenciones: relación entre la comprensión lectora y la teoría de la mente
}

Carlos Ramos Morales ${ }^{2}$

cramos@userena.cl

Nina María Crespo Allende ${ }^{3}$

ncrespo@ucv.cl

\section{RESUMEN}

La teoría de la mente se relaciona con la capacidad que tenemos de representarnos intenciones y creencias de los otros. Esta habilidad implica el desarrollo de teorías en una recursividad cada vez más compleja y es fundamental para la comunicación oral (Meltzoff, 1999). La literatura sitúa su desarrollo entre los 6 y 7 años, justo cuando empieza de manera formal el aprendizaje de la lectoescritura. A partir de allí, el presente trabajo tiene como propósito examinar la relación entre la teoría de la mente y la comprensión de textos narrativos en niños normales que cursan tercer año de educación básica. Para evaluar la teoría de la mente se diseñó un instrumento basado en las tareas clásicas de falsas creencias de segundo orden (Sullivan y Tager-Flusberg, 1999 y Perner y Wimmer, 1985), y para la evaluación de la comprensión de lectura de texto narrativo se creó un instrumento sustentado en el modelo de comprensión de van Dijk y Kinstch (1983).

Palabras clave

Teoría de la mente, comprensión lectora.

\section{ABSTRACT}

The theory of mind is associated with the ability all human beings have to represent to themselves the others' intentions and beliefs. This ability implies the development of increasingly complex theories and it is essential to oral communication (Meltzoff, 1999). Research on this subject places its development between the ages of 6 and 7 , just at the onset of formal learning of reading and writing. From there, the objective of this paper is to explore the relationship between theory of mind and the comprehension of narrative texts in normal third graders. To evaluate the theory of mind, an instrument based on the classic tasks of second-order false beliefs (Sullivan \& Tager-Flusberg, 1999, and Perner \& Wimmer, 1985) was designed, and to evaluate the comprehension of the narrative text an instrument supported by the van Dijk \& Kintsch's (1983) model of comprehension was created.

\section{KEYWORDS}

Theory of mind, reading comprehension.

1 Trabajo de investigación financiado por el proyecto Fondecyt 1070333.

2 Dr. en Lingüística. Académico del Departamento de Educación Universidad de La Serena, Chile. Proyecto de Investigación: FONDECYT 1070333.

3 Dra. en Lingüística. Académica del Instituto de Literatura y Ciencias del Lenguaje, Pontificia Universidad Católica de Valparaíso, Chile. Proyecto de Investigación: fondecyt 1070333. 


\section{Introducción}

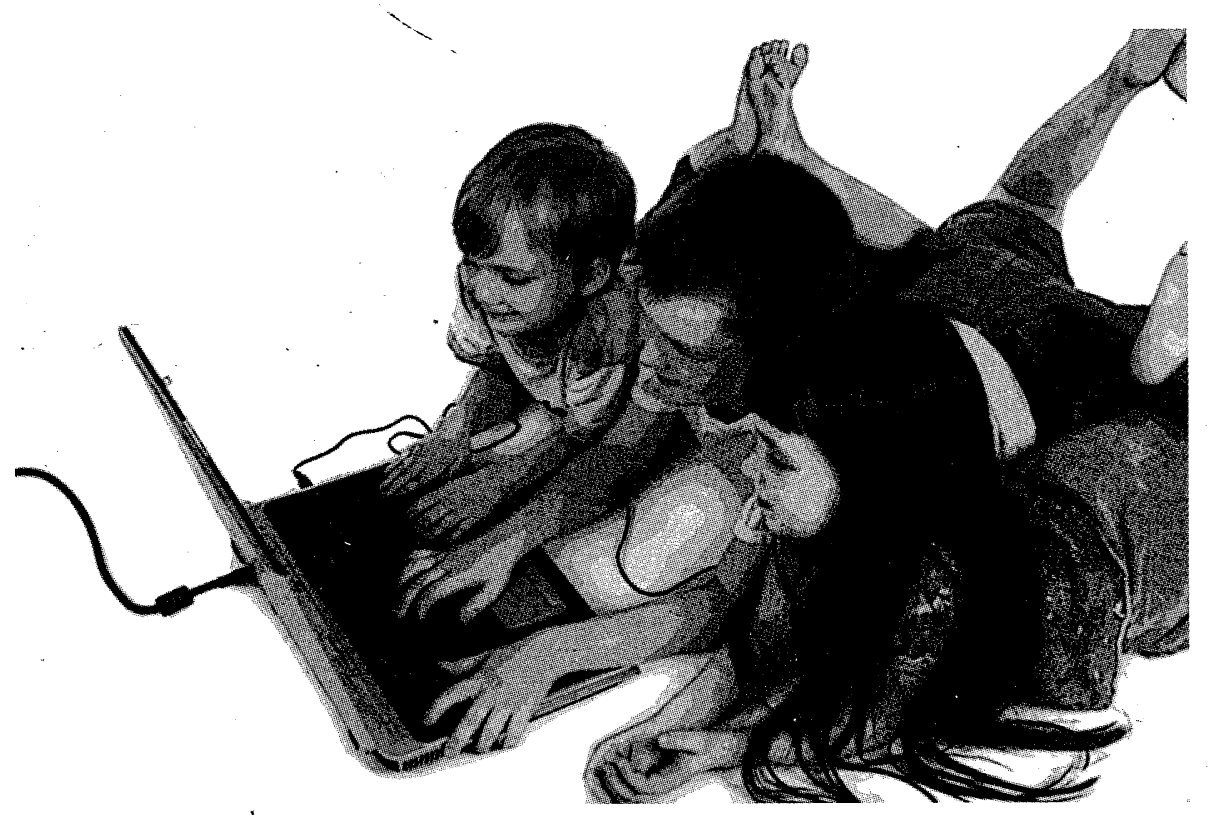

$\mathrm{E}$ 1 concepto de teoría de la mente es un constructo teórico que se ha propuesto para explicar la capacidad o mecanismo cognitivo (Happé, 1998) que poseen los seres humanos para atribuir estados mentales independientes de sí mismos y de los demás (BaronCohen, Leslie y Frith, 1985). Las operaciones específicas de esta capacidad están orientadas hacia la representación, interpretación y predicción del comportamiento humano en términos de estados mentales, tales como creencias, deseos, intenciones, emociones, imaginación, etc. En otras palabras, tal como señala Baron-Cohen (2001), poseer teoría de la mente es ser capaz de reflexionar e inferir el contenido de la propia mente y el contenido de la mente de los demás. Así, Bosacki y Astington (1999) establecen que los sujetos pueden atribuir estados mentales a otras personas para predecir su comportamiento, para saber qué pensarán, para comprender lo que quieren decir y cuál es la intención de lo que dicen.

Esta capacidad para "leer la mente" (Happé, 1998) permite que las personas comprendan el comportamiento social. De esta forma, se puede explicar por qué las personas normales pueden concebir el mundo social como un sistema organizado, con sentido, perceptible y predecible. Esta con- cepción del mundo social favorece y hace posible la interacción con otras personas, y a su vez permite que se establezcan formas de comportamiento diferentes a la interacción que se tiene con los objetos. La interacción social es una interacción de mentes, mediada a través del lenguaje (Happé, 1998; Astington, 1998, y Nelson, 2005). En ese sentido, según Baron-Cohen (1990), la teoría de la mente es fundamental para la comunicación. Para ello, los participantes deben tener en cuenta el conocimiento previo, las presuposiciones e intenciones que el otro posee al momento de comunicar.

Como ya se ha señalado, la teoría de la mente consiste en la comprensión del comportamiento de las personas en términos de estados mentales. Astington (1998) afirma que los estados mentales son los pensamientos -creencias, deseos, intenciones- y que no se pueden observar. Sin embargo, éstos se infieren a partir de la observación de las acciones de las personas y, especialmente, de la comprensión de los enunciados lingüísticos que expresan esos estados mentales en actos de habla -las creencias como aserciones, los deseos como peticiones, las intenciones como promesas, etc.

El desarrollo de la teoría de la mente se inicia a edades muy tempranas. Este inicio, se- 
gún Astington (1998), se manifiesta cuando los niños empiezan a tener conciencia de que ellos mismos y de que las otras personas tienen mente, y de que en la mente se encuentrañ los estados mentales. Dicha conciencia les permite comprender progresivamente que los estados mentales determinan las causas del comportamiento humano. Este logro incluye la adquisición de dos tipos de conocimientos implícitos: en primer lugar, los niños deben darse cuenta de que los estados mentales son representaciones o metarrepresentaciones (Leslie, 1994) que solo existen en la mente, y en segundo lugar, que esos estados son construidos por la mente y permiten a las personas relacionarse de formas particulares con el mundo.

Leslie (1994), desde una posición modular, sostiene que los niños poseen capacidades innatas para comprender el mundo, tanto para comprender los objetos físicos, sus propiedades y sus relaciones, así como para comprender al otro y establecer relaciones interpersonales. Para el logro de tales comprensiones, los niños construyen representaciones mentales de los objetos tal como son en la realidad y construyen metarrepresentaciones para representar estados mentales o actitudes proposicionales como pensar, intentar, desear, creer (Happé, 1998 y Rivière y. Núñez, 2001). Nelson (2005) afirma que las representaciones mentales de este tipo solo son posibles mediante el uso del lenguaje.

También sostiene Nelson que el desarrollo más importante, el que tiene más impacto en el funcionamiento social y cognitivo, es la adquisición del sistema lingüístico y su uso como un sistema representacional. Así, los niños pueden utilizar el lenguaje para la reflexión, para la transferencia de conocimientos, la imaginación, el recuerdo, las explicaciones y otras funciones sociales, culturales y cognitivas. Cuando los niños empiezan a construir metarrepresentaciones aprenden a comprender que las palabras tales como pensar, conocer y recordar se refieren a estados mentales, y que esas palabras generan proposiciones que representan el contenido mental de los otros.

Algunas investigaciones revelan que el desarrollo de la teoría de la mente, a nivel de habilidades mentales más complejas, alcanza su madurez alrededor de los 11 años de edad (Happé, 1994).

\section{Comprensión de textos escritos}

Van Dijk y Kintsch (1983) sostienen que los textos escritos constituyen una estructura formada por una serie de ideas o proposiciones unidas por medio de relaciones semánticas. Algunas de estas relaciones se encuentran expresamente señaladas en el texto escrito, mientras que otras deben ser establecidas por el lector mediante procesos inferenciales (Parodi, 1999). La estructura de los textos contempla la microestructura y la macroestructura (Kintsch, 1998; van Dijk, 1983; van Dijk y Kintsch, 1983, y Parodi, 1999). La microestructura está compuesta por el conjunto de relaciones proposicionales de elementos menores o de coherencia local. La macroestructura corresponde al tipo de estructura más abstracta que representa el significado global o coherencia global del texto. Lo anterior se organiza en una estructura esquemática denominada superestructura. Cada texto posee una superestructura particular que organiza en forma jerarquizada sus distintos fragmentos en función de la intención del autor.

\section{Cuando los niños empiezan a construir metarrepresentaciones aprenden a comprender que las palabras tales como pensar, conocer y recordar se refieren a estados mentales, y que esas palabras generan proposiciones que representan el contenido mental de los otros.}

La comprensión ocurre cuando los lectores construyen una representación mental del significado del texto, teniendo como base su estructura y la superestructura que la sostiene. Dicha representación implica la construcción de tres niveles de representación: representación superficial, representación del texto base y 
representación del modelo de situación. La representación superficial del texto es el nivel más básico de la comprensión. Es un registro breve de los estímulos gráficos exactos, ya seàn palabras u oraciones, que se han leído más recientemente (Kintsch, 1998 y Parodi, 1999). La representación del texto base es una copia semántica equivalente a la estructura superficial del texto. El lector construye este nivel de comprensión aplicando estrategias cognitivas que reducen la microestructura para formar la macroestructura del texto (Kintsch, 1998 y Parodi, 1999). El modelo de situación es la representación mental final del proceso de comprensión. Kintsch (1998) sostiene que este nivel de comprensión es la comprensión propiamente tal, ya que refleja la incorporación del texto base al conocimiento previo del lector en una estrecha relación con sus propósitos y actitudes.

El aprendizaje de la comprensión empieza en los primeros grados escolares. Una vez que el proceso de decodificación se ha automatizado, la mayoría de los niños empieza a comprender textos escritos que se corresponden con su nivel de desarrollo. Tienden a tener más facilidad para comprender textos narrativos que otros tipos de textos. Autores como Snow (2002), Cain (1996) y Fitzgerald (1991) establecen que esta mayor facilidad de comprensión se debe a que los textos narrativos relatan historias que contienen información sobre episodios, personajes y eventos estrechamente relacionados con la vida real. Además, los niños tienen conocimiento de la superestructura narrativa desde la infancia temprana (Nelson, 1996 y Condemarín, 1989). Stein y Albro (1997) sostienen que esta estructura se lo-

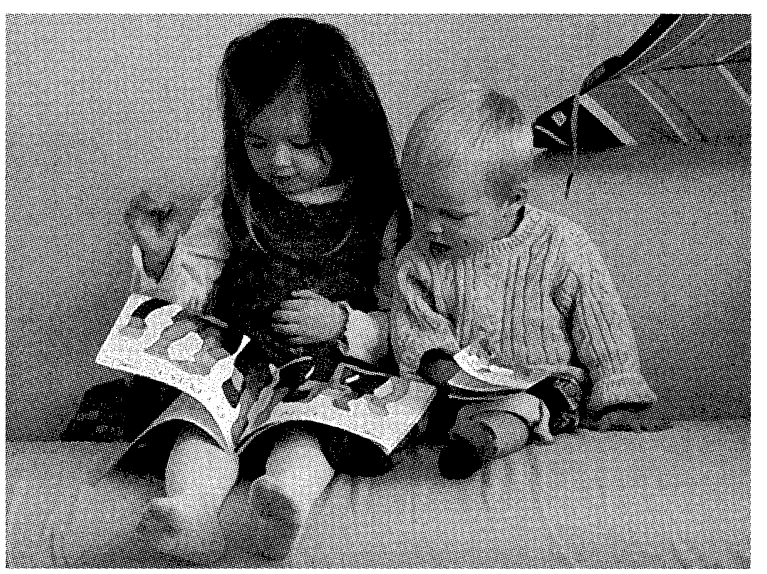

gra a los tres años de edad como resultado de la comprensión que tienen los niños de las intenciones que motivan las acciones humanas. En consecuencia, este conocimiento contribuiría a activar una gran cantidad de conocimientos cuando se enfrentan a la lectura de textos de este tipo.

Es necesario destacar que no sólo el conocimiento de la superestructura favorece la comprensión de los textos escritos. Es necesario que los niños pongan en juego otra serie de habilidades. Entre ellas, las habilidades para realizar inferencias que se requieren para construir la micro y macroestructura textual y la habilidad para monitorear su propia comprensión, entre otras (Perffeti, Landi y Oakhill, 2005).

En cuanto a la relación de teoría de la mente y comprensión de narraciones, Nelson (2005) sostiene que la comprensión de narraciones requiere del mismo tipo de representaciones mentales que son requeridas en la teoría de la mente. Cuando los niños escuchan una historia requieren hacer uso del lenguaje como una representación personal interna de la narración escuchada. A partir de ello, se puede concluir que esta capacidad de comprensión presente en la oralidad puede ser transferida a la comprensión de textos narrativos escritos, como uno de los factores que puede incidir en su desarrollo.

\section{Metodología}

\subsection{Objetivo}

Examinar la relación entre teoría de la mente y la comprensión de textos narrativos en niños normales que cursan tercer año de educación básica.

\subsection{Sujetos}

La muestra estuvo compuesta inicialmente por 40 escolares y fue ampliada a 94 niños y niñas de tercer año básico pertenecientes a colegios subvencionados y municipales de las regiones de $\mathrm{Co}^{-}$ quimbo y Valparaíso, Chile. Sus edades estaban comprendidas entre los 8 y 9 años. Ninguno de los escolares presentaba antecedentes de dificultades de aprendizaje u otros trastornos del desarrollo. 


\subsection{Instrumentos de evaluación}

\section{a) Evaluación de la teoría de la mente}

Para evaluar la teoría de la mente se diseñó un instrumento (Test том) compuesto de cuatro historias. Tres historias clásicas de falsas creencias de segundo orden (Perner y Wimmer, 1985 y Sullivan y Tager-Flusberg, 1999) y una historia de persuasión (Happé, 1998). Cada historia evalúa tres niveles de comprensión de los estados mentales de los personajes: nivel de comprensión de falsas creencias de primer orden, nivel de comprensión explícita de segundo orden y nivel de comprensión inferencial de segundo orden.

A continuación se indica para cada nivel de comprensión el tipo de habilidades requeridas:

a) Nivel de comprensión de falsas creencias de primer orden. Este nivel implica la ejecución de habilidades referidas a la capacidad de inferir el estado mental o creencia de otra persona, la comprensión literal de la historia y la capacidad para justificar una respuesta.

b) Nivel de comprensión explicita de segundo orden. Este nivel comprende las habilidades para identificar lo que no sabe una persona que sabe otra, la comprensión de esas relaciones y la capacidad para justificar una respuesta.

c) Nivel de comprensión inferencial de segundo orden. Este nivel de comprensión implica las habilidades para reconocer lo que una persona piensa sobre las creencias o sentimientos de otra persona, la comprensión literal de segundo orden y la capacidad para justificar una respuesta.

El instrumento se aplicó en forma individual. Cada historia se narró a los niños por partes, según niveles de comprensión, en formato PowerPoint. Después de narrar cada parte se plantearon las preguntas que requerían la elaboración de la respuesta por parte de los niños. Las respuestas se registraron tal como fueron expresadas por los evaluados y se les asignó un puntaje de acuerdo a su nivel de precisión en la descripción de los estados mentales de los personajes.

\section{b) Evaluación de la comprensión de lectura}

La comprensión de lectura se evaluó mediante una prueba creada de acuerdo al modelo de van Dijk y Kintsch (1983). Consta de dos historias con una extensión de 168 palabras cada una. Las historias corresponden a cuentos populares indios. Se adecuaron sus características de legibilidad para escolares de tercer año básico (Alliende, 1994). Cada una evalúa tres niveles de comprensión: nivel de comprensión de la microestructura textual, nivel de comprensión de la macroestructura textual y nivel de comprensión de la superestructura textual. La prueba tiene un total de 20 preguntas. Para cada texto se propone el mismo número de preguntas que miden las mismas habilidades para cada nivel de comprensión.

La comunicación humana se
basa sobre todo en un cálculo
de las intenciones del otro en un
contexto, es decir, en la teoría
de la mente, y que el lenguaje
es un estímulo más que aporta
a la comprensión de un mensaje
ostensiblemente transmitido.

A continuación se indica para cada nivel de comprensión el tipo de habilidades requeridas:

a) Nivel de comprensión de la microestructura textual. Este nivel implica la habilidad para comprender relaciones de coherencia referencial y causal que se expresan en oraciones adyacentes.

b) Nivel de comprensión de la macroestructura textual. Este nivel implica la habilidad para comprender la relación entre ideas parciales y globales del texto.

c) Nivel de comprensión de la superestructura textual. Este nivel implica el conocimiento de la estructura textual y sus categorías internas para ejecutar el proceso de comprensión.

La prueba de comprensión de lectura se aplicó en forma colectiva y en formato de papel y lápiz. La técnica de evaluación es de tipo prueba objetiva con ítems de elección múltiple. Cada 
pregunta se acompaña de cuatro alternativas de respuesta y se admite sólo una respuesta correcta. La elección de esta técnica permite obtener una valoración más precisa de la comprensión, dado que los alumnos de tercer año básico aún se encuentran en la etapa inicial del aprendizaje de las habilidades más complejas de la lectoescritura.

\section{Análisis de los resultados}

A continuación se presentan los resultados de acuerdo al análisis estadístico de los datos realizado por Montenegro y Pérez (2007). El apartado presentará inicialmente el nivel de logro de los sujetos en cada instrumento, luego dará cuenta de la confiabilidad de los mismos y finalmente presentará la relación entre ambas pruebas.

\subsection{Nivel de logro de los sujetos en ambos instrumentos}

El nivel de logro de las pruebas se evaluó estimando el porcentaje de respuestas correctas. De esta forma, se puede apreciar en el gráfico 1 que en el Test Tom, en la medida en que aumenta la dificultad, disminuye el porcentaje de respuestas correctas.

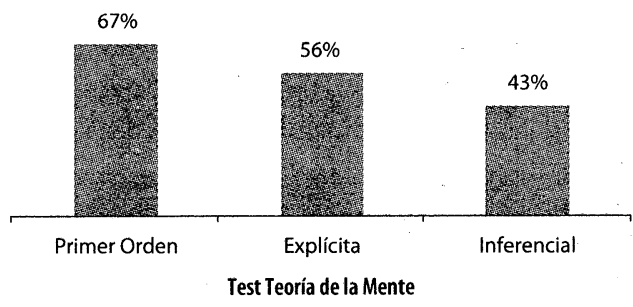

Gráfico 1: Test том.

Así, se observa que el $67 \%$ de las respuestas fueron correctas para el nivel de comprensión de falsas creencias de primer orden (N1), $1.55 \%$ para el nivel de comprensión de falsas creencias de segundo orden (N2) y $43 \%$ para el nivel comprensión inferencial de segundo orden (N3). En términos generales la prueba tuvo un $55 \%$ de respuestas correctas.

En el gráfico 2 se puede apreciar que en el test de comprensión lectora los tres niveles evaluados registran niveles de logros distintos:

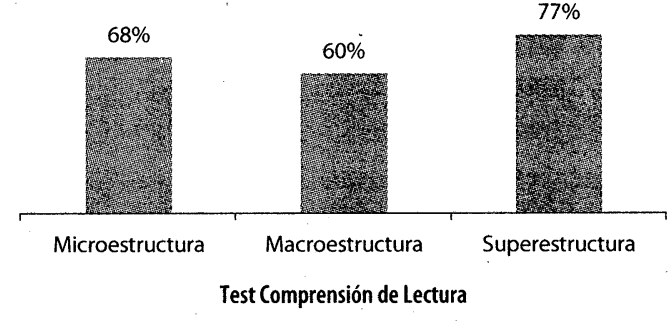

Gráfico 2: Test de comprensión de lectura.

De esta manera, el mayor porcentaje de logro se observa a nivel de la superestructura con un $77 \%$, seguido por el nivel de la microestructura con $68 \%$ y, finalmente, el más difícil resultó ser el de la macroestructura con un logro del $60 \%$. En términos generales la prueba tuvo un $68 \%$ de respuestas correctas.

\subsection{Confiabilidad de los instrumentos}

Para medir la confiabilidad de los instrumentos se utilizó el coeficiente Alpha de Cronbach y se observó que ambas pruebas eran confiables. Así, se observó que el test de teoría de la mente tiene una confiabilidad muy alta $(0,89)$ y el test de comprensión lectora tiene una confiabilidad alta $(0,72)$, de acuerdo a Ruiz (1988).

\subsection{Relación de la teoría de la mente y la comprensión lectora}

El propósito fundamental del presente estudio es examinar la relación entre la teoría de la mente y la comprensión de textos narrativos en niños normales que cursan tercer año de educación básica. Para esto, se utilizó una síntesis de cada test a través de los puntajes totales obtenidos por los sujetos en cada uno de ellos. El análisis exploratorio gráfico de la relación existente entre los puntajes totales de ambas pruebas revela que existe una relación entre ellas, la que tiene un comportamiento no lineal.

En el gráfico 3 se puede observar el diagrama de dispersión para los puntajes de la prueba Tом y la prueba de comprensión de lectura. Se muestra la curva ajustada a los datos por medio del suavizador Lowess (Cleveland; 1979).

Además, la reláción entre ambas variables revela una correlación positiva significativa (rpb 
$=0,21 ; \mathrm{p}<0,05)$, que indica que el desarrollo de ciertas habilidades cognitivas es indispensable para el desarrollo de la lectura.

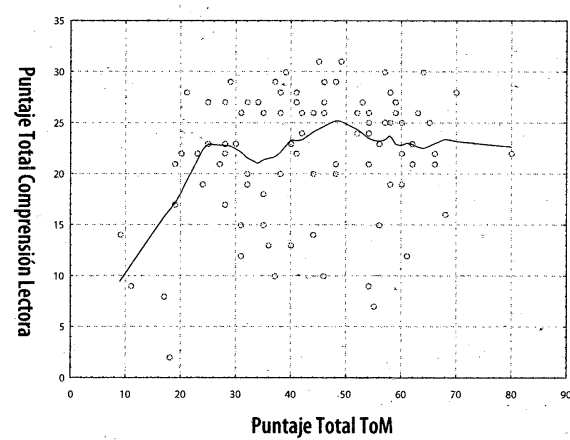

Gráfico 3: Diagrama de dispersión том y comprensión lectora.

\section{Conclusiones}

En primer lugar, es necesario hacer hincapié en que ambos instrumentos han resultado altamente confiables y que, por lo tanto, estos datos, aunque preliminares, resultan válidos para realizar un primer acercamiento a la relación entre la comprensión lectora y la teoría de la mente.

Respecto del grado de dificultad de las pruebas, cabe señalar que la de la teoría de la mente resultó más difícil que la de comprensión lectora. Dichos resultados no parecerían coincidir con la impresión de que la comprensión de la lengua escrita resulta más difícil que la de la lengua oral implícita en la medición de la том. Estos resultados apuntarían a señalar que el cálculo de los estados mentales pareciera ser, para un niño de 8 años, una habilidad de mayor exigencia inferencial que la comprensión de lo narrativo, aunque ésta implique la puesta en juego de una habilidad de codificación lingüística más sofisticada.

No obstante, dicha diferencia en el nivel de logro pudo haber tenido una causal en el formato de las pruebas. En otras palabras, la mayor o menor dificultad de cada prueba pudo haber dependido de su diseño, ya que, mientras la prueba том exigía una elicitación de la respuesta elaborada lingüísticamente, la prueba de comprensión sólo exigía el reconocimiento de la alternativa de respuesta correcta. Por este motivo, es necesario volver a medir ambas variables con pruebas de formato más homogéneas para poder concluir respecto de su mayor grado de dificultad relativa.

Ahora bien, en la relación de la teoría de la mente con la capacidad de comprensión lectora, vemos una correlación baja pero significativa, lo que estaría indicando que la capacidad de comprender el mundo intencional de los otros y de metarrepresentarse sus estados mentales sería una de las condiciones fundamentales para lograr una comprensión de los contenidos de los textos narrativos. Este vínculo pareciera estar acorde con aquel supuesto teórico sostenido -entre otros- por la teoría de la relevancia (Wilson y Sperber, 2002), la cual sostiene que la comunicación humana se basa sobre todo en un cálculo de las intenciones del otro en un contexto, es decir, en la teoría de la mente, y que el lenguaje es un estímulo más que aporta a la comprensión de un mensaje ostensiblemente transmitido.

Por otra parte, la conexión de la comprensión lectora con la teoría de la mente pareciera corroborarse con los casos de hiperlexia registrados en niños autistas (Lopera y Uribe, 1998). Entre otros problemas, estos sujetos no son capaces de armar una teoría de la mente adecuada (Baron-Cohen, 2001) y autores como Lopera y Uribe (1998) señalan que, los que aprenden a leer, logran sólo una automatización del proceso de decodificación grafema-fonema, pero son incapaces de comprender lo que leen, fenómeno denominado biperlexia.

A partir de estos hallazgos es posible señalar que si bien la decodificación (basada en habilidades de naturaleza fonológica) es fundamental en la lectura de los primeros años escolares, para lograr un nivel de comprensión lectora adecuado el niño debe tener una madurez cognitiva referida no sólo al conocimiento del mundo concreto, sino a aquel relacionado con representaciones y metas de otros seres humanos. En otras palabras, como Catherine Nelson (2005) señala, es necesario utilizar el lenguaje completo como un sistema representacional. Ó

\section{Bibliografía}

Alliende, F. (1994), La legibilidad de los textos, Santiago, Andrés Bello.

Astington, J.W. (1998), El descubrimiento infantil de la mente, Madrid, Morata. 
Baron-Cohen, S. (2001), "Theory of Mind and Autism: A review", en International review of research in mental retardation, núm. 23, pp. 169-184.

- Leslie, A. M., y Frith, U. (1985), "Does the Autistic Child Have a «Theory of Mind»?", en Cognition, núm. 21, pp. 37-46.

Bosacki, S. y Astington, J. M. (1999), “Theory of Mind in Preadolescents: Relations Between Language and Theory-of-Mind Development", en Development Psychology, vol. 5, núm. 535, pp. 1311-1320.

Cain, K. (1996), "Store Knowledge and Comprehension Skill”, en Cornoldi, C. y Oakhill, J. (edits.), Reading Comprehension Difficulties: Processes and Intervention, Mahwah, NJ, Erlbaum, pp. 167-192.

Cleveland, W. S. (1979), "Robust Locally Weighted Regression and Smoothing Scatterplots", en Journal of the American Statistical Association, núm. 74, pp. 829-836.

Condemarín, M. (1989), Lectura temprana (jardin infantil y primer grado), Santiago, Andrés Bello.

Fitzgerlad, J. (1991), "Investigaciones sobre el texto narrativo: Implicancias didácticas", en Denise Ruth, K. (comp.), El texto narrativo, Buenos Aires, Aique, pp. 15-42.

Happé, F. (1998), Introducción al autismo, Madrid, Alianza.

Kintsch, W. (1998), Comprehension: A paradigm for cognition, New York, Cambridge University Press.

Leslie, A. (1994), “томм, тову and Agency: Core Architecture and Domain Specific", en Hirschfeld, L. A. y Gelman, S. A. (edits), Mapping the Mind Domain Specificity in Cognition and Culture, New York, Cambridge University Press, pp. 119-148.

Meltzoff, A. (1999), "Origins of Theory of Mind, Cognition, and Communication", en Journal of Communication Disorders, núm. 32, pp. 251-269.

Montenegro, C y Pérez, L. (2007), Análisis Estadistico I: Test teoría de la mente - Test comprensión lectora proyecto Fondecyt 1070333 [Documento interno].
Lopera, F, y Uribe, C.(1998), "Presentación de caso: Hiperlexia y autismo", Iatreia, vol. 2, núm 11, pp. 77-84.

Nelson, K. (2005), "Language Pathways into the Community of Minds", en Astington, J. W. y Baird, J. (edits.), Why Language Matters to Theory of Mind, Cambridge University Press, pp.26-49.

Parodi, G. (1999), Relaciones entre lectura y escritura: una perspectiva cognitiva discursiva. Bases teóricas y antecedentes empiricos, Valparaíso, Ediciones Universitarias de Valparaíso de la Universidad Católica de Valparaíso.

Perffeti, Ch.; Landi, N. y Oakhill, J. (2005), "The Acquisition of Reading Comprehension Skill”, en Snowling, M. y Hulme, C. (edits.), The science of reading: $A$ bandbook, Oxford, Blackwell.

Perner, J. y Wimmer, H. (1985), “"John Thinks that Mary Thinks that...»: Attribution of Second-Order Beliefs by 5 to 10 -year-old Children", en Journal of Experimental Child Psychology, núm. 39, pp. 437-471.

Riviere, A. y Núñez, M. (2001), La mirada mental, Buenos Aires, Aique.

Ruiz Bolívar, C. (1998), Instrumentos de Investigación Educativa, CIDEG, Barquisimeto, Lara, Venezuela.

Snow, C. (2002), Reading for Understanding: Toward a Research and Development Program in Reading Comprehension, Santa Monica, CA, Rand Corporation.

Stein, N. y Albro, E. (1997), “The Emergence of Narrative Understanding: Evidence for Rapid Learning in Personally Relevant Contexts", en Contemporary Issues in Education, núm. 60, pp. 83-98.

Sullivan, K. y Tager-Flusberg, H. (1999), "Second Order Belief Attribution in William Syndrome: Intact or Impaired?", en American Journal of Mental Retardation, núm. 104, pp. 523-532.

van Dijk, T. y Kintsch, W. (1983), Strategic discourse comprehension, New York, Academia Press.

Wilson, D. y Sperber, D. (2002), "Relevance Theory", en Horn, L. y Ward, G. (edits.), Handbook of Pragmatics Psycholinguistics, Oxford, Blackwell, pp. 34-78. 